\title{
CHARACTERIZATION OF ELECTRICAL TRANSPORT IN LSMO WITH ENHANCED TEMPERATURE OF METAL-INSULATOR TRANSITION
}

\author{
Vladimír Štrbík — Štefan Chromik *
}

\begin{abstract}
We have studied $\mathrm{La}_{0.67} \mathrm{Sr}_{0.33} \mathrm{MnO}_{3}$ (LSMO) thin films with temperature of metal-insulator $\left(T_{\mathrm{MI}}\right)$ transition enhanced to above $400 \mathrm{~K}$, and we estimated characteristic electrical transport mechanisms for these films. We have fitted the measured resistivity vs. temperature $\rho(T)$ dependence in a wide temperature range $4-500 \mathrm{~K}$ using different mechanisms of the electrical transport in different parts of $\rho(T)$. In addition to the narrow temperature range around $T_{\mathrm{MI}}$ very well agreement was found. We found out that the Debye's temperature was also increased $\left(\Theta_{D} \approx 840 \mathrm{~K}\right)$ probably due to the change in crystallization of LSMO films.
\end{abstract}

K e y w or d s: LSMO thin film, electrical transport, Debye temperature

\section{INTRODUCTION}

Perovskite manganites exhibit strongly correlated magnetic and electrical transport properties. At a certain temperature $\mathrm{T}_{\mathrm{MI}}$ manganites undergo a metal-insulator transition and a ferromagnetic (FM) to paramagnetic (PM) transition as well. At temperatures higher than $\mathrm{T}_{\mathrm{MI}}$ the resistance temperature dependence behaves like insulators and magnetically they are in a PM state. For temperatures below $\mathrm{T}_{\mathrm{MI}}$ they behave like metals and are ferromagnetic (ferromagnetic-metal state). The metalinsulator transition is very simply observable as a resistance peak in the $\rho(T)$ dependence and $T_{M I}$ corresponds to the temperature of the maximal resistance. Among the manganites the $\mathrm{La}_{0.67} \mathrm{Sr}_{0.33} \mathrm{MnO}_{3}$ (LSMO) is the most attractive material because of its high Curie temperature. For high quality $\mathrm{La}_{1-x} \mathrm{Sr}_{x} \mathrm{MnO}_{3}(x=0.3$ and 0.4$)$ single crystals the Curie temperature $T_{C} \approx 370 \mathrm{~K}$ was referred [1]. Recently Sadock et al [2] published their results that the Curie temperature can reach a value up to $650 \mathrm{~K}$ for a multilayer $\mathrm{LSMO} / \mathrm{BaTiO}_{3}$ heterostructure.

We have prepared the LSMO thin films with the enhanced value of $\mathrm{T}_{\mathrm{MI}}>400 \mathrm{~K}$ whereby detailed X-ray analyzes showed changes in crystallization of these films [3]. While the films with $\mathrm{T}_{\mathrm{MI}}<370 \mathrm{~K}$ crystallize in a pseudo-cubic form, the films with $\mathrm{T}_{\mathrm{MI}}>370 \mathrm{~K}$ have a proper microstructure with distorted orthorhombic unit cells. Therefore, it was interesting to examine whether the new crystallization has an influence on the LSMO electrical transport properties.

\section{EXPERIMENTAL}

The LSMO films were deposited using on-axis dc magnetron sputtering from a stoichiometric $\mathrm{La0} .{ }_{67} \mathrm{Sr}_{0.33} \mathrm{MnO}_{3}$ ceramic target onto one-side polished $\mathrm{MgO}(001)$ substrates. The deposition was performed in an $\operatorname{Ar}(80 \%)+$ $\mathrm{O}_{2}(20 \%)$ atmosphere at a total pressure of $65 \mathrm{~Pa}$. The substrate was heated to $800{ }^{\circ} \mathrm{C}$ during the deposition. The thickness of the LSMO films was close to $100 \mathrm{~nm}$ and the film growth rate was around $12 \mathrm{~nm} / \mathrm{min}$. The LSMO films were subsequently in-situ annealed in $\mathrm{O}_{2}\left(10^{4} \mathrm{~Pa}\right)$ at $800{ }^{\circ} \mathrm{C}$ for an hour [3]. For the $\rho(T)$ measurement a standard four-point method was applied. The electrodes on the samples were formed with a silver paint and the sample was heated in a calibrated furnace.

\section{RESULTS AND DISCUSSION}

Structural and compositional analyses (Auger electron spectroscopy, X-ray diffraction, Transmission electron microscopy) indicate that the LSMO films are stoichiometric, single phase and epitaxial [3,4], in spite of some nonideal crystallographic matching of the LSMO film with the $\mathrm{MgO}$ substrate. The main attribute of the LSMO films - the enhanced $\mathrm{T}_{\mathrm{MI}}$ — we were able to prepare reproducibly. The experimental resistivity vs. temperature $\rho(T)$ dependence of the LSMO film on the $\mathrm{MgO}$ substrate is shown in Fig. 1. The resistivity peak is relatively broad and the maximal $\rho$ is at $\mathrm{T}_{\mathrm{MI}}=400 \mathrm{~K}$. The $\rho(T)$ dependence measured in the temperature range $4-$ $500 \mathrm{~K}$ can be divided into several parts. The approximate boundaries between the parts are denoted by black points with corresponding temperatures $T_{0}-T_{3}$ (in Fig. 1). Each

\footnotetext{
* Institute of Electrical Engineering, SAS, Dúbravská cesta 9, 84104 Bratislava, Slovakia, elekstrb@savba.sk
} 


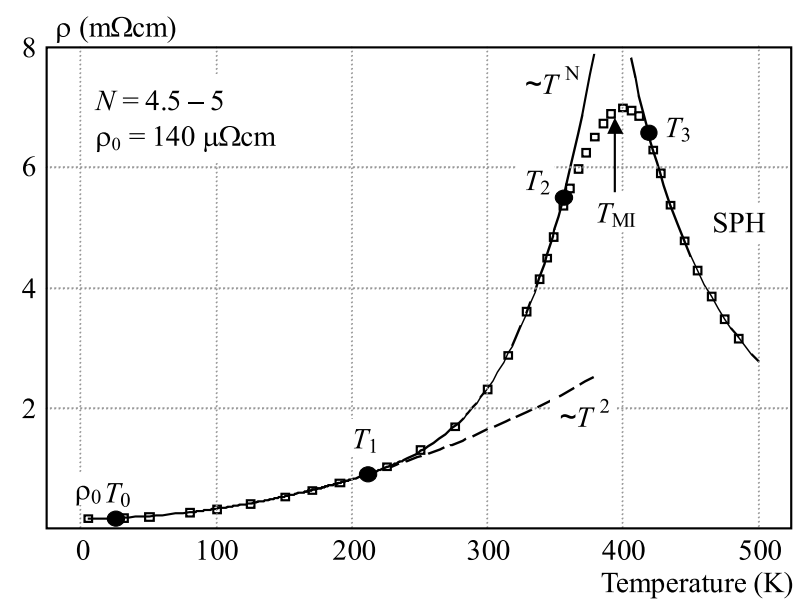

Fig. 1. The measured resistivity vs. temperature dependence of LSMO film on $\mathrm{MgO}$ substrate. The black points determinate the parts of dependence with own characteristic scattering mechanism (more in text)

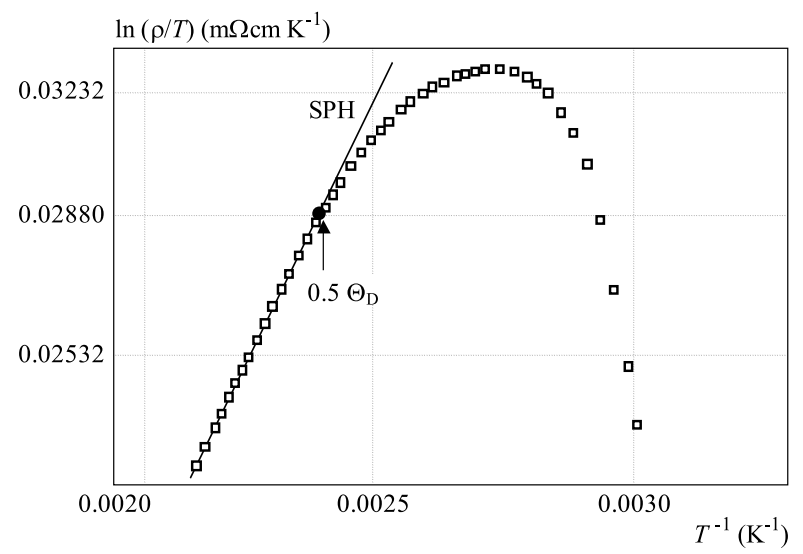

Fig. 2. The plot of $\ln (\rho / T)$ vs $T^{-1}$ dependence. Full line represents the SPH model

part of the $\rho(T)$ is characterized by a specific scattering mechanism.

The resistivity in the lowest temperature range (below $T_{0} \approx 20 \mathrm{~K}$ ) exhibits essentially constant value of the residual resistivity $\rho_{0}$, which is determined mainly by temperature independent scattering on impurities, defects, grain boundaries or domain walls. The typical value of $\rho_{0}$ is in the range of $100-200 \mu \Omega \mathrm{cm}$ and it is about two times higher than $\rho_{0}$ of the single crystal [1] or of the high quality LSMO thin films [5]. In the temperature range $T_{0}-T_{1}$ (range $20-220 \mathrm{~K}$ ) the resistivity data follows the $T^{2}$ dependence. Such a dependence of the resistivity is frequently observed and it is ascribed to the intrinsic property of La-based manganites [6] to electron-electron scattering [1] or to one-magnon scattering [7].

Above the temperature $T_{1}(\approx 220 \mathrm{~K})$ the resistivity increases much more than $T^{2}$ dependence (see Fig. 1) and the temperature dependence of higher power is evident. The double exchange theory which describes the ferromagnetic ground state offers the $T^{4.5}$ dependence of the resistivity due to an the electron-magnon scattering [8] and some supporting role in this temperature range plays the electron-phonon scattering with the $T^{5}$ dependence [5]. Recently we have introduced the modified polynomial fit formula $\rho=\rho_{0}+\rho_{2} T^{2}+\rho_{N}\left(T-T_{N}\right)^{N}$, where $\rho_{0}, \rho_{2}, \rho_{N}$ and $T_{N}$ are fitting parameters and $4.5 \leq N \leq 5$ [9]. This formula fits well the experimental dependence from $4 \mathrm{~K}$ up to the temperature $T_{2}\left(\approx 355 \mathrm{~K}\right.$ ) (Fig. 1, full line for $T<\mathrm{T}_{\mathrm{MI}}$ ) and has significantly increased temperature range of validity in comparison to previous approaches $[1,5-7]$, where authors fitted their experimental dependences only to temperatures $250-300 \mathrm{~K}$. The temperature $T_{2}$ appears significant because at this temperature the LSMO starts to undergo from the ferromagnetic to the paramagnetic state and simultaneously to the metal-insulator transition. This transition can be described only qualitatively. As it was mentioned above the electron-magnom and electron-phonon scatterings are responsible for the rapid increase of the resistivity in the temperature range $T_{1}-T_{2}$. For further temperature increase (above $T_{2}$ ) the phonon scattering becomes stronger and a polaron conductivity is created. Just above $T_{2}$ the polarons are heavy free carriers scattered by phonons and give positive $\mathrm{d} \rho / \mathrm{d} T$ [6]. At the temperature $\mathrm{T}_{\mathrm{MI}}$ the phonon scattering is so strong that the polaron carriers are localized and further electrical transport for the temperature above $\mathrm{T}_{\mathrm{MI}}$ is possible only through a thermally activated hopping mechanism with negative $\mathrm{d} \rho / \mathrm{d} T[6]$. The transport in high-temperature range (above $T_{3} \approx 420 \mathrm{~K}$ ) is well described by small polaron hopping $(\mathrm{SPH})$ theory $\rho=\rho_{h} \operatorname{Texp}\left(E_{h} / k_{B} T\right)[10]$, where $E_{h}$ is the hopping energy, $k_{B}$ is Boltzman's constant and $\rho_{h}$ is a resistivity coefficient. The full line in the temperature range above $\mathrm{T}_{\mathrm{MI}}$ in Fig. 1 represents the SPH model. One can see that the presented transport mechanisms well describe the $\rho(T)$ of the LSMO thin films with the enhanced $\mathrm{T}_{\mathrm{MI}}$.

The SPH model is valid for temperatures $T>\Theta_{D} / 2$, where $\Theta_{D}$ is Debye's temperature [11]. Our detailed graph (Fig. 2) indicates that the validity of the $\mathrm{SPH}$ model (full line) finishes at the temperature $T \sim 420 \mathrm{~K}$ eg $\Theta_{D} \approx 840 \mathrm{~K}$. This is significantly higher than the reported one before $\sim 500 \mathrm{~K}[11,12]$. The increased value of $\Theta_{D}$ can be evoked by the change in the crystallization of the LSMO thin films with $\mathrm{T}_{\mathrm{MI}}>370 \mathrm{~K}$ and also the correlation between $\Theta_{D}$ and $T_{M I}$ should be studied in more details.

\section{CONCLUSIONS}

We have prepared $\mathrm{La}_{0.67} \mathrm{Sr}_{0.33} \mathrm{MnO}_{3}$ (LSMO) thin films with the enhanced temperature of metal-insulator transition $\left(\mathrm{T}_{\mathrm{MI}} \geq 400 \mathrm{~K}\right.$ ). The resistivity $v$ s temperature $\rho(T)$ dependence in the temperature range $4-500 \mathrm{~K}$ was characterized by different transport mechanisms in different parts of $\rho(T)$. The temperature independent scattering, electron-electron, one-magnon, electron-magnon 
and electron-phonon scattering mechanisms were considered to describe the $\rho(T)$ dependence in the temperature range $0<T \leq 355 \mathrm{~K}$. In the temperature interval $355 \mathrm{~K}<T<420 \mathrm{~K}$ the LSMO overcomes ferromagnetic-paramagnetic and metal-insulator transitions via polarons conductivity. For temperatures higher than $\mathrm{T}_{\mathrm{MI}}$ the polarons become localized and further transport is performed only by thermal activated hopping. The transport above $420 \mathrm{~K}$ is described by the model of small polaron hopping and from this dependence we have estimated the Debye's temperature $\Theta_{D} \approx 840 \mathrm{~K}$.

\section{Acknowledgment}

This publication is the result of the project implementation: CENTE II, ITMS code 26240120019, supported by the Research \& Development Operational Programme funded by the ERDF.

\section{REFERENCES}

[1] URUSHIBARA, A.-MORITOMO, Y.-ARIMA, T.-ASAMITSU, A.-KIDO, G.-TOKURA, Y.: Insulator-Metal Transition and Giant Magnetoresistance in $\mathrm{La}_{1-x} \mathrm{Sr}_{x} \mathrm{MnO}_{3}$, Physical Review B 51 (1995), 14103-14109.

[2] SADOC, A.-MERCEY, B.-SIMON, CH.-GREBILLE, D.PRELLIER, W.-LEPETIT, M.-B.: Large Increase of the Curie Temperature by Orbital Ordering Control, Physical Review Letters 104 (2010), 046804-1-4.

[3] CHROMIK, S̆.-ŠTRBÍK, V.-DOBROČKA, E.-DUJAVOVÁ, A.—REIFFERS, M.-LIDAY, J.—ŠPANKOVÁ, M. : Significant Increasing of Onset Temperature of FM Transition in LSMO Thin Films, paper prepared for publishing.

[4] ŠPANKOVÁ, M.-CHROMIK, Š.—VÁVRA, I.—SEDLÁČKOVÁ, K.-LOBOTKA, P.-LUCAS, S.-STANČEK, S. : Epitaxial LSMO Films Grown on MgO Single Crystalline Substrates, Applied Surface Science 253 (2007), 7599-7603.

[5] BOSCHKER, H.-HUIJBEN, M. VAILIONIS,-A. VERBEECK,-J.-van AERT, S.-LUYSBERG, M.-BALS, S.van TENDELOO, G.-HOUWMAN, E. P.-KOSTER, G.BLANK, D. H. A.-RIJNDERS, G.: Optimized Fabrication of High-Quality $\mathrm{La}_{0.67} \mathrm{Sr}_{0.33} \mathrm{MnO}_{3}$ Thin Films Considering All Essential Characteristics, Journal of Physics D: Applied Physics 44 (2011), 205001-1-9.

[6] JEFFREY SNYDER, G.-HISKES, R.-DiCAROLIS, S.BEASLEY, M. R.-GEBALLE, T. H.: Intrinsic Electrical Transport and magnetic Properties of $\mathrm{La}_{0.67} \mathrm{Ca}_{0.33} \mathrm{MnO}_{3}$ and $\mathrm{La}_{0.67} \mathrm{Sr}_{0.33} \mathrm{MnO}_{3}$ MOCVD Thin Films and Bulk Material, Physical Review B 53 (1996), 14434-14444.

[7] JAIME, M.-LIN, P.-SALAMON, M. B.-HAN, P. D. : LowTemperature Electrical Transport and Double Exchange in
$\mathrm{La}_{0.67}(\mathrm{~Pb}, \mathrm{Ca})_{0.33} \mathrm{MnO}_{3}$, Physical Review B 58 (1998), R5901-R5104.

[8] KUBO, K.-OHATA, N.: A Quantum Theory of Double Exchange, Journal of the Physical Society of Japan 33 (1972), $21-32$.

[9] ŠTRBÍK, V.-CHROMIK, Š- ŠPANKOVÁ, M. : LSMO Thin Films with High $\mathrm{T}_{\mathrm{MI}}$ for Bolometric Applications, paper prepared for publishing.

10] EMIN, D.-HOLSTEIN, T. : Studies of Small-Polaron Motion IV. Adiabatic Theory of the Hall Effect, Annals of Physics $\mathbf{5 3}$ (1969), 439-520.

[11] VENKATAIACH， G.-PRASAD， V.-VENUGOPAL REDDY, P.: Influence of A-Site Cation Mismatch on Structural, Magnetic and Electrical Properties of Lanthanum Manganites, Journal of Alloys and Compounds 429 (2007), 1-9.

12] SCHRIFFER, P.-RAMIREZ, A. P.-BAO, W.-CHEONG, S.-W.: Low Temperature Magnetoresistance and the Magnetic Phase Diagram of $\mathrm{La}_{1-x} \mathrm{Ca}_{x} \mathrm{MnO}_{3}$, Physical Review Letters 75 (1995), 3336-3339.

Received 12 December 2011

Vladimír Štrbík (RNDr, CSc) graduated from the Faculty of Natural Sciences of Comenius University in Experimental Physics with specialization on Physics of condensed matter and acoustics in 1977. He started with Department of Cryoelectronics in Institute of Electrical Engineering (IEE), Slovak Academy of Sciences (SAS), Bratislava, Slovakia (1977) and he continues to work at IEE SAS up to date. In 1987, he received his $\mathrm{PhD}(\mathrm{CSc})$ degree in field of superconducting cryoelectronic devices and circuits. He works at IEE SAS as scientific worker mainly in field of electrical and magnetic properties of superconducting thin films, structures, devices and circuits. He has solved many domestic and international scientific projects as principal investigator or co-worker. His results have been presented in more than 70 articles in scientific periodicals and in more than 50 contributions at international conferences.

Štefan Chromik (Ing, DrSc), graduated from the Faculty of Electrical Engineering at Slovak University of Technology, Bratislava, Slovakia, in 1973. He started with Department of Cryoelectronics in Institute of Electrical Engineering, Slovak Academy of Sciences (IEE SAS), Bratislava, Slovakia (1973) and he continues to work at IEE SAS up to date. Now he is head of Department of Cryolectronics. He works at IEE SAS as scientific worker mainly in field of preparation of superconducting, ferromagnetic and dielectric thin films. He has solved many domestic and international scientific projects as principal investigator or co- worker. He authored or co-authored more than 110 papers in scientific journals (cited more than 270 times) and 8 patents. 